\title{
HOLOMORPHIC ONE-FORMS ON VARIETIES OF GENERAL TYPE
}

\author{
By CHRISTOPHER D. HACON ${ }^{1}$ AND SÁNDOR J. KOVÁCS ${ }^{2}$
}

\begin{abstract}
It has been conjectured that varieties of general type do not admit nowhere vanishing holomorphic one-forms. We confirm this conjecture for smooth minimal varieties and for varieties whose Albanese variety is simple.
\end{abstract}

(C) 2005 Elsevier SAS

RÉSUMÉ. - Selon une conjecture connue, les variétés de type général n'admettent pas de 1-formes holomorphes partout non-nulles. Nous vérifions cette conjecture pour les variétés lisses minimales et pour celles dont la variété d'Albanese est simple.

(C) 2005 Elsevier SAS

\section{Introduction}

The impact of zeros of vector fields on the geometry of the underlying variety has been studied extensively, cf. $[6,4,5,9,8,10,3,1,2]$. For instance, it is known that the existence of a nowhere zero vector field on a compact complex manifold implies that all of its characteristic numbers vanish.

Carrell asked whether something similar is implied by the existence of a nowhere vanishing holomorphic one-form. He proved that this is the case for surfaces, namely if $S$ is a compact complex surface admitting a nowhere vanishing holomorphic one-form, then $c_{1}(S)^{2}$ and $c_{2}(S)$ are zero [7]. On the other hand, he also gave an example of a threefold $X$, a $\mathbb{P}^{1}$-bundle over an abelian surface, for which $c_{1}(X)^{3} \neq 0$. This suggests that one needs to treat varieties with negative Kodaira dimension differently.

Having to pay attention to the Kodaira dimension of the variety makes it natural to approach the problem from the point of view of classification theory and first restrict to the case of minimal varieties. For a minimal variety $X, K_{X}$ is nef, therefore $c_{1}(X)^{\operatorname{dim} X} \neq 0$ is equivalent to $K_{X}^{\operatorname{dim} X}>0$ which is equivalent to $X$ being of general type.

We are also led to varieties of general type via a different path. If $X$ admits a nowhere vanishing holomorphic one-form, then [16, Theorem 3.1] implies that for generic $\mathscr{P} \in \operatorname{Pic}^{0}(X)$, one has $H^{i}\left(X, \Omega_{X}^{j} \otimes \mathscr{P}\right)=0$ for all $i, j$. In particular, $\chi\left(X, \omega_{X}\right)=0$. On the other hand, when $X$ is of maximal Albanese dimension (i.e., $\left.\operatorname{dim} X=\operatorname{dim} \operatorname{alb}_{X}(X)\right)$ and $\operatorname{Alb}(X)$ is simple, then $\mathrm{X}$ is a variety of general type if and only if $\chi\left(X, \omega_{X}\right)>0$.

All of these considerations naturally lead to the following conjecture.

\footnotetext{
${ }^{1}$ The author was partially supported by NSA grant MDA904-03-1-0101 and by a grant from the Sloan Foundation.

${ }^{2}$ The author was partially supported by NSF grant DMS-0092165 and a Sloan Research Fellowship.
} 
CONJECTURE 1.1. - Let $X$ be a smooth projective variety of general type. Then $X$ does not admit a nowhere vanishing holomorphic one-form.

Once we focus on varieties of general type, restricting to the case of minimal varieties is the right thing to do according to a conjecture of Carrell:

CONJECTURE 1.2 (Carrell). - Let $X$ be a smooth projective variety of general type. If $X$ admits a nowhere vanishing holomorphic one-form, then $X$ is minimal.

Remark 1.2.1. - This is known for surfaces and using the classification of extremal contractions one can easily see that it also holds for threefolds. This was explicitly checked in [24, Lemma 2.1].

Conjecture 1.1 has been confirmed for canonically polarized varieties (i.e., whose canonical divisor is ample) in [29] and for threefolds in [24].

An immediate consequence of this conjecture is that a variety of general type does not admit any smooth morphisms onto an abelian variety. For other applications the reader is referred to [29].

In this article we first prove Conjecture 1.1 for smooth minimal varieties.

THEOREM 1.3. - Let $X$ be a smooth projective minimal variety of general type. Then $X$ does not admit a nowhere vanishing holomorphic one-form.

This completely confirms Conjecture 1.1 assuming Conjecture 1.2. Using Remark 1.2.1 this also gives a new proof of the threefold case [24, Theorem 1].

Using different methods than the ones used to prove Theorem 1.3, we also confirm Conjecture 1.1 for varieties whose Albanese variety is simple.

THEOREM 1.4. - Let $X$ be a smooth projective variety of general type. If its Albanese variety is simple, then $X$ does not admit a nowhere vanishing holomorphic one-form.

Definitions and notation 1.5. - Let $f: X \rightarrow Y$ be a proper morphism. A line bundle $\mathscr{L}$ on $X$ is called $f$-nef if $\operatorname{deg}\left(\left.\mathscr{L}\right|_{C}\right) \geqslant 0$ for every proper curve $C \subseteq X$ such that $f(C)$ is a point. $\mathscr{L}$ is called $f$-big if $\operatorname{rank} f_{*} \mathscr{L}^{m}>c \cdot m^{n}$ where $c>0$ is a constant and $n$ is the dimension of the general fibre of $f$. If $Y=\operatorname{Spec} k$ for an algebraically closed field $k$, then $f$-nef (respectively $f$-big) is simply called nef (respectively big). For $\theta \in H^{0}\left(X, \Omega_{X}\right), Z(\theta)$ denotes the zero locus of $\theta$. Let $\mathscr{F}$ be a torsion-free sheaf on $X$ and $\iota: U \hookrightarrow X$ the locus where $\mathscr{F}$ is locally free. Then $\widehat{S}^{m}(\mathscr{F})$ denotes the reflexive hull of the $m$ th symmetric power of $\mathscr{F}$, i.e., $\widehat{S}^{m}(\mathscr{F})=\iota_{*} S^{m}\left(\left.\mathscr{F}\right|_{U}\right)$.

\section{Smooth minimal models}

The main goal of this section is to prove the following.

THEOREM 2.1. - Let $Y$ be a projective variety with only rational singularities of dimension $n$, and let $\phi: X \rightarrow Y$ be a resolution of singularities of $Y$. Let $\phi^{\#} \Omega_{Y}=\operatorname{im}\left[\phi^{*} \Omega_{Y} \rightarrow \Omega_{X}\right]$. Assume that there exists a $\theta \in H^{0}\left(X, \phi^{\#} \Omega_{Y}\right)$ such that the zero locus of $\theta$ is empty. Then for any ample line bundle $\mathscr{L}$ on $Y, H^{n}(Y, \mathscr{L})=0$.

Before we can prove this theorem we need some preparation.

Let $X$ be a smooth variety of dimension $n$. Let $\Phi$ be the functor of regular functions and $\Psi$ the functor of Kähler differentials, i.e., $\Phi_{X}=\mathscr{O}_{X}$ and $\Psi_{X}=\Omega_{X}$. Then any $\theta \in H^{0}\left(X, \Omega_{X}\right)$ induces a morphism $\theta_{X}: \Phi_{X} \rightarrow \Psi_{X}$. In fact it induces a morphism $\theta_{X_{i}}: \Phi_{X_{i}} \rightarrow \Psi_{X_{i}}$ via pull

$4^{\text {e }}$ SÉRIE - TOME $38-2005-\mathrm{N}^{\circ} 4$ 
back for every $X_{i}$ that admits a morphism, $\phi_{i}: X_{i} \rightarrow X$, to $X$. In other words $\theta$ induces a natural transformation from $\Phi$ to $\Psi$ in the category of $X$-schemes. Then by [23, 2.6, 2.9] there exists a functorially defined $\underline{Q}_{\theta_{X}}^{r} \in \mathrm{Ob}(D(X))$ for all $r \geqslant-1$ such that for every $p \in \mathbb{N}$ there exists a distinguished triangle,

$$
\underline{\mathfrak{Q}}_{\theta_{X}}^{p-1} \rightarrow \Omega_{X}^{p} \rightarrow \underline{\mathfrak{Q}}_{\theta_{X}}^{p} \stackrel{+1}{\longrightarrow} .
$$

Furthermore, $\underline{\mathfrak{Q}}_{\theta_{X}}^{r} \simeq 0$ if $r>n-1$ and $\underline{\mathfrak{Q}}_{\theta_{X}}^{n-1} \simeq \omega_{X}$.

Suppose $Z(\theta)$ is empty. Then by [14, Appendix B.3.4] the Koszul complex

$$
0 \rightarrow \mathscr{O}_{X} \stackrel{\wedge \theta}{\longrightarrow} \Omega_{X}^{1} \stackrel{\wedge \theta}{\longrightarrow} \Omega_{X}^{2} \stackrel{\wedge \theta}{\longrightarrow} \cdots \stackrel{\wedge \theta}{\longrightarrow} \Omega_{X}^{n-1} \stackrel{\wedge \theta}{\longrightarrow} \Omega_{X}^{n} \rightarrow 0
$$

induced by taking the wedge product with $\theta$ is exact. Let $\mathscr{E}^{-1}=0$, and

$$
\mathscr{E}^{i}=\operatorname{ker}(\wedge \theta): \Omega_{X}^{i} \rightarrow \Omega_{X}^{i+1}
$$

for $i=1, \ldots, n-1$. Then $\underline{\mathfrak{Q}}_{\theta_{X}}^{r} \simeq \mathscr{E}{ }^{r+1}$ for $r=0, \ldots, n-2$. In particular $\underline{\mathfrak{Q}}_{\theta_{X}}^{0} \simeq \mathscr{O}_{X}$.

Next, results regarding the generalized De Rham complexes are summarized in the following theorem.

THEOREM 2.2 [11] [17, III.1.12, V.3.3, V.3.6, V.5.1]. - For every complex scheme $Y$ of dimension $n$ there exists an $\underline{\Omega}_{Y} \in \mathrm{Ob}\left(D_{\mathrm{filt}}(Y)\right)$ with the following properties:

(2.2.1) Let $\phi .: X . \rightarrow Y$ be any hyperresolution of $Y$. Then $\underline{\Omega}_{Y} \simeq R \phi_{. *} \Omega_{X}$.

(2.2.2) The definition is functorial, i.e., if $\phi: X \rightarrow Y$ is a morphism of complex schemes, then there exists a natural map $\phi^{*}$ of filtered complexes

$$
\phi^{*}: \underline{\Omega}_{Y} \rightarrow R \phi_{*} \underline{\Omega}_{X} .
$$

Furthermore, $\underline{\Omega}_{Y} \in \operatorname{Ob}\left(D_{\text {filt,coh }}^{b}(Y)\right)$ and if $\phi$ is proper, then $\phi^{*}$ is a morphism in $D_{\text {filt,coh }}^{b}(Y)$.

(2.2.3) Let $\Omega_{Y}$ be the usual De Rham complex of Kähler differentials considered with the "filtration bête". Then there exists a natural map of filtered complexes

$$
\Omega_{Y} \rightarrow \underline{\Omega}_{Y}
$$

and if $Y$ is smooth, it is a quasi-isomorphism.

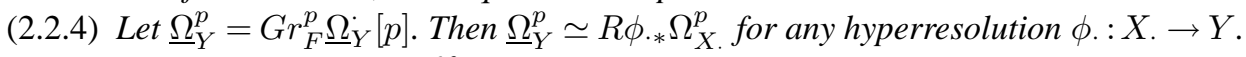

(2.2.5) If $Y$ is projective and $\mathscr{L}$ is an ample line bundle on $Y$, then

$$
\mathbb{H}^{q}\left(Y, \underline{\Omega}_{Y}^{p} \otimes \mathscr{L}\right)=0 \quad \text { for } p+q>n .
$$

To extend the definition of $\underline{Q}_{\theta_{X}}^{p}$ to singular varieties we need the following.

Lemma 2.3. - Let $\phi .: X . \rightarrow Y$ be a hyperresolution of $Y$. Let $\phi^{\#} \Omega_{Y}$ be defined as in (2.1). Let $\theta \in H^{0}\left(X_{0}, \phi^{\#} \Omega_{Y}\right)$ and $\theta_{X_{i}}: \mathscr{O}_{X_{i}} \rightarrow \Omega_{X_{i}}$ be the morphism induced by the section $\theta$. Then $R \phi \cdot \mathfrak{Q}_{\theta_{X}}^{r}$ is independent of the hyperresolution chosen. 
Proof. - Let $\alpha$ be a morphism of hyperresolutions.

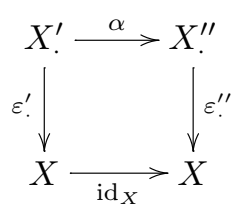

Then by $[23,4.1]$ there exists a commutative diagram:

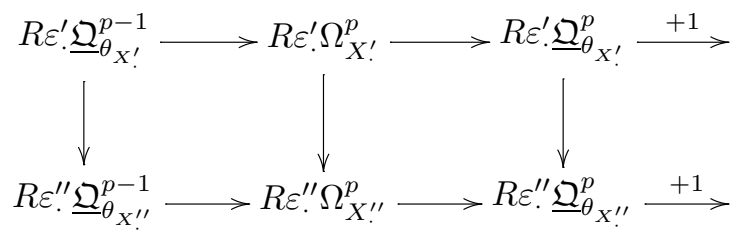

Now $R \varepsilon^{\prime} \Omega_{X^{\prime}}^{p} \simeq \underline{\Omega}_{Y}^{p} \simeq R \varepsilon^{\prime \prime} \Omega_{X^{\prime \prime}}^{p}$ by (2.2.4), and the statement follows from [11, 2.1.4] and ( $\star$ ) by descending induction on $p$.

DEFinition 2.4. - Let $Y$ be a variety of dimension $n$. Let $\phi .: X . \rightarrow Y$ be a hyperresolution of $Y$ and let $\theta \in H^{0}\left(X_{0}, \phi^{\#} \Omega_{Y}\right)$. We define $\underline{\mathfrak{Q}}_{\theta_{Y}}^{r}=R \phi_{\cdot *} \underline{\mathfrak{Q}}_{\theta_{X}}^{r}$ for $r \geqslant-1$. By the lemma, this is independent of the hyperresolution chosen, in particular if $Y$ is smooth, it agrees with the previous definition of $\underline{\mathfrak{Q}}_{\theta_{Y}}^{r}$.

Proof of Theorem 2.1. - By $(\star)$ there exists a distinguished triangle,

$$
\underline{\mathfrak{Q}}_{\theta_{Y}}^{p-1} \rightarrow \underline{\Omega}_{Y}^{p} \rightarrow \underline{\mathfrak{Q}}_{\theta_{Y}}^{p} \stackrel{+1}{\longrightarrow}
$$

for every $p \in \mathbb{N}$, so by (2.2.5),

$$
\mathbb{H}^{n-p}\left(Y, \underline{\mathfrak{Q}}_{\theta_{Y}}^{p} \otimes \mathscr{L}\right) \rightarrow \mathbb{H}^{n-(p-1)}\left(Y, \underline{\mathfrak{Q}}_{\theta_{Y}}^{p-1} \otimes \mathscr{L}\right)
$$

is surjective for all $p$, and then

$$
\mathbb{H}^{0}\left(Y, \underline{\mathfrak{Q}}_{\theta_{Y}}^{n} \otimes \mathscr{L}\right) \rightarrow \cdots \rightarrow \mathbb{H}^{n}\left(Y, \underline{\mathfrak{Q}}_{\theta_{Y}}^{0} \otimes \mathscr{L}\right)
$$

is also surjective. Now $\mathbb{H}^{0}\left(Y, \underline{\mathfrak{Q}}_{\theta_{Y}}^{n} \otimes \mathscr{L}\right)=0$ since $\underline{\mathfrak{Q}}_{\theta_{Y}}^{n}=0$, so we obtain that

$$
\mathbb{H}^{n}\left(Y, \underline{\mathfrak{Q}}_{\theta_{Y}}^{0} \otimes \mathscr{L}\right)=0 .
$$

On the other hand, the previous observation in the case $Z(\theta)=\emptyset,(2.2 .2),(2.2 .3),(\star \star)$ and $[23,4.1]$ implies that the following diagram is commutative:

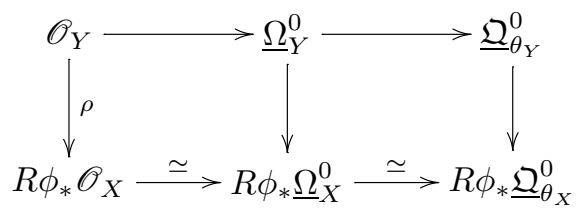

$4^{\text {e }}$ SÉRIE - TOME $38-2005-\mathrm{N}^{\circ} 4$ 
Now $\rho$ has a left inverse, and hence in turn the morphism $\mathscr{O}_{Y} \rightarrow \underline{Q}_{\theta_{Y}}^{0}$ has a left inverse. Finally that implies that $H^{n}(Y, \mathscr{L}) \rightarrow \mathbb{H}^{n}\left(Y, \underline{\mathfrak{Q}}_{\theta_{Y}}^{0} \otimes \mathscr{L}\right)=0$ is injective.

The following lemma is a simple corollary of the Basepoint-free theorem. This fact is well known to experts in higher dimensional birational geometry, but since it seems not that well known otherwise we include a proof for the convenience of the reader.

LEMMA 2.5. - Let $X$ be a projective variety with rational Gorenstein singularities and assume that $\omega_{X}$ is nef and big. Then $\bigoplus_{m=0}^{\infty} H^{0}\left(X, \omega_{X}^{m}\right)$ is a finitely generated $k$-algebra and $Y=\operatorname{Proj}\left(\bigoplus_{m=0}^{\infty} H^{0}\left(X, \omega_{X}^{m}\right)\right)$ has rational Gorenstein singularities and $\omega_{Y}$ is an ample line bundle.

Proof. $-\bigoplus_{m=0}^{\infty} H^{0}\left(X, \omega_{X}^{m}\right)$ is a finitely generated $k$-algebra by [20, (3.11)]. The Basepointfree theorem [20, (3.3)] (cf. [26]) implies that for $m \gg 0, \omega_{X}^{m}$ is generated by global sections. Then the morphism given by the global sections of $\omega_{X}^{m}$ maps to $Y$, it is independent of $m \gg 0$ and is its own Stein factorization. I.e., there exist a birational morphism $\phi: X \rightarrow Y$ and ample line bundles $\mathscr{M}_{m}$ on $Y$ such that $\omega_{X}^{m}=\phi^{*} \mathscr{M}_{m}$. Hence

$$
\omega_{X} \simeq \omega_{X}^{m+1} \otimes\left(\omega_{X}^{m}\right)^{-1} \simeq \phi^{*}\left(\mathscr{M}_{m+1} \otimes \mathscr{M}_{m}^{-1}\right)
$$

and so $\phi_{*} \omega_{X} \simeq \mathscr{M}_{m+1} \otimes \mathscr{M}_{m}^{-1}$ is an ample line bundle. In particular, it is reflexive. $Y$ is normal and $\phi$ is birational, so $\phi_{*} \omega_{X}$ agrees with $\omega_{Y}$ on an open subset $U$ such that $\operatorname{codim}(Y \backslash U, Y) \geqslant 2$. They are both reflexive, so $\omega_{Y} \simeq \phi_{*} \omega_{X}$ is an ample line bundle.

This implies that $Y$ has canonical singularities and then it has rational singularities by [13] (cf. [22]). In particular, $Y$ is Cohen-Macaulay and combined with $\omega_{Y}$ being a line bundle that implies that $Y$ is Gorenstein.

We are now ready to prove our main theorem.

Proof of Theorem 1.3. - Consider the Albanese morphism of $X$,

$$
\operatorname{alb}_{X}: X \rightarrow \operatorname{Alb}(X)
$$

and the natural morphism from $X$ to its canonical model

$$
\phi: X \rightarrow Y=\operatorname{Proj}\left(\bigoplus_{m=0}^{\infty} H^{0}\left(X, \omega_{X}^{m}\right)\right) .
$$

By a result of Reid (cf. [19, (8.1)]) alb ${ }_{X}$ factors through $\phi$. Therefore, using the notation of (2.1), $H^{0}\left(X, \Omega_{X}\right)=H^{0}\left(X, \phi^{\#} \Omega_{Y}\right)$. By (2.5) $Y$ has rational singularities and $\omega_{Y}$ is an ample line bundle, so (1.3) follows from (2.1) and the fact that $H^{n}\left(Y, \omega_{Y}\right) \neq 0$.

\section{Varieties whose Albanese variety is simple}

We are going to study the Albanese morphism of $X$ and employ different strategies depending on whether it is surjective or not.

Case I. $-\operatorname{alb}_{X}$ is not surjective.

PROPOSITION 3.1. - Let $Z \subsetneq A$ be a proper closed subvariety of the abelian variety $A$. If $A$ is simple, then for every holomorphic one-form $\theta \in H^{0}\left(A, \Omega_{A}^{1}\right),\left.\theta\right|_{Z}$ has a non-empty zero set. 
Proof. - Let $\mathcal{W} \subset H^{0}\left(A, \Omega_{A}^{1}\right)$ be the set of those holomorphic one-forms $\theta \in H^{0}\left(A, \Omega_{A}^{1}\right)$ such that $\left.\theta\right|_{Z}$ vanishes at some point $z \in Z$. It is easy to see that $\mathcal{W}$ is closed and so it suffices to show that $\mathcal{W}$ is dense in $H^{0}\left(A, \Omega_{A}^{1}\right)$.

Let $r=\operatorname{dim} Z$ and let $Z_{0}$ be the set of smooth points of $Z$. For any $z \in Z_{0}$, one has that the tangent space $T_{z}(Z) \cong \mathbb{C}^{g-r} \subset T_{z}(A) \cong H^{0}\left(A, \Omega_{A}^{1}\right)^{\vee} \cong \mathbb{C}^{g}$. Let $\mathcal{Z} \subset \mathbb{P}^{g-1}$ be the closure of the image $\mathcal{Z}_{0}$ of the corresponding projective bundle $\mathcal{P}:=\mathbb{P}\left(T\left(Z_{0}\right)^{\perp}\right)$ under the corresponding map. One sees that if $\mathcal{Z}=\mathbb{P}^{g-1}$, then $\mathcal{W}$ is dense in $H^{0}\left(A, \Omega_{A}^{1}\right)$.

Suppose that $\mathcal{Z} \neq \mathbb{P}^{g-1}$, i.e., $\operatorname{dim} \mathcal{Z}<g-1$. Let $p \in \mathcal{Z}$ be a general point, then $\operatorname{dim} \mathcal{Z}<g-1$ implies that the corresponding fiber $\mathcal{P}_{p}$ is positive dimensional. Consider now the projection $\pi: \mathcal{P} \rightarrow Z_{0}$ and the subvariety $Z_{p}$ given by the closure of $\pi\left(\mathcal{P}_{p}\right) \cap Z_{0} \subset A$; one has

$$
\operatorname{dim} Z_{p}=g-1-\operatorname{dim} \mathcal{Z}>0 .
$$

For general $x \in Z_{p}$, one has for $L_{p}$ the line corresponding to $p$ that $L_{p} \subset T_{x}(Z)^{\perp}$ and so $T_{x}\left(Z_{p}\right) \subset T_{x}(Z) \subset H_{p}:=L_{p}^{\perp}$. It follows that $Z_{p}$ generates a proper abelian subvariety $A_{p} \subsetneq A$. This is a contradiction, so $\mathcal{W}=H^{0}\left(A, \Omega_{A}^{1}\right)$.

Corollary 3.2. - Let $X$ be a projective variety and $\alpha: X \rightarrow A$ a morphism to a simple abelian variety. If $Z:=\alpha(X) \neq A$, then every holomorphic one-form

$$
\theta \in \alpha^{*} H^{0}\left(A, \Omega_{A}^{1}\right) \subset H^{0}\left(X, \Omega_{X}^{1}\right)
$$

has a non-empty zero set.

Case II. - alb $_{X}$ is surjective.

Notation 3.3. - Let $X$ be a projective variety and let $\alpha: X \rightarrow A$ be a surjective morphism to an abelian variety. Let $\Delta \subset A$ be the locus where $\alpha$ is not smooth.

PROPOSITION 3.4. - Under the assumptions of (3.3) assume that $\Delta$ contains an irreducible divisor $D$ of general type. Then every holomorphic one-form $\theta \in \alpha^{*} H^{0}\left(A, \Omega_{A}^{1}\right) \subset H^{0}\left(X, \Omega_{X}^{1}\right)$ has a non-empty zero set.

Proof. - Consider $\mathcal{W} \subset H^{0}\left(A, \Omega_{A}^{1}\right)$ the set of those holomorphic one-forms $\theta \in H^{0}\left(A, \Omega_{A}^{1}\right)$ such that $\alpha^{*} \theta \in H^{0}\left(X, \Omega_{X}^{1}\right)$ vanishes at some point $x \in X$. As above, $\mathcal{W}$ is closed and so it suffices to show that it is dense.

Consider $D^{0} \subset D$ a (non-empty) open set such that for all $z \in D^{0}$ there is a point $x \in X_{z}$ with $\operatorname{rank}\left(d \alpha_{x}\right)=g-1$ (cf. [18, III.10.6]) and $D$ is smooth at $z$. Let $x_{1}, \ldots, x_{g}$ be local coordinates of $A$ at $z$ such that $D$ is defined by $x_{g}=0$ and $\theta=\theta_{z} \in H^{0}\left(A, \Omega_{A}^{1}\right)$ such that $\theta(z)=d x_{g}$. Then, $\theta$ spans the subspace $T_{z}(D)^{\perp} \subset H^{0}\left(A, \Omega_{A}^{1}\right)$ and $\left.\theta\right|_{D}$ vanishes at $z$ and $\alpha^{*} \theta$ vanishes at some point $x \in X$ such that $a(x)=z$ (in fact at any such point with $\operatorname{rank}\left(d \alpha_{x}\right)=g-1$ ). Since $D$ is of general type, by [15] (cf. [25, (3.9)]), its Gauss map is generically finite and so one sees that the set $\left\{\theta_{z} \mid z \in D^{0}\right\} \subset \mathcal{W}$ is dense in $H^{0}\left(A, \Omega_{A}^{1}\right)$. (Reasoning as in the previous proposition, we have that $\mathcal{P} \cong Z_{0}$ and $\mathcal{P} \rightarrow \mathbb{P}^{g-1}$ is generically finite and so it is dominant.)

PROPOSITION 3.5. - Under the assumptions of (3.3) assume that there exists a positive integer $m$ such that $\alpha_{*}\left(\omega_{X / A}^{m}\right)$ is big. Then $\alpha$ is not smooth in codimension one, i.e., $\Delta$ contains a divisor.

Proof. - Since $\alpha_{*}\left(\omega_{X / A}^{m}\right)$ is big, for any ample line bundle $\mathscr{H}$ on $A$ there exists an integer $a>0$ such that $\widehat{S}^{a}\left(\alpha_{*}\left(\omega_{X / A}^{\otimes m}\right)\right) \otimes \mathscr{H}^{-1}$ is big. Let $m_{k}: A_{k} \simeq A \rightarrow A$ be multiplication by an integer $k$, so $m_{k}$ is an étale map such that $m_{k}^{*} \mathscr{H}=\left(\mathscr{H}_{k}\right)^{k}$ with $\mathscr{H}_{k}$ an ample line bundle on $A_{k}$.

$4^{\mathrm{e}}$ SÉRIE - TOME $38-2005-\mathrm{N}^{\circ} 4$ 
Let $g=\operatorname{dim} A, r=\operatorname{dim} X-\operatorname{dim} A$, and $k=3 r(g-1) m a$. Further let $H_{k}$ be a divisor on $A_{k}$ such that $\mathscr{O}_{A}\left(H_{k}\right) \simeq \mathscr{H}_{k}$ and finally let

$$
\alpha^{\prime}: X^{\prime}:=X \times_{A} A_{k} \rightarrow A_{k}=A .
$$

Then

$$
m_{k}^{*}\left(\widehat{S}^{a}\left(\alpha_{*}\left(\omega_{X / A}^{m}\right)\right) \otimes \mathscr{H}^{-1}\right)=\widehat{S}^{a}\left(\alpha^{\prime}{ }_{*}\left(\omega_{X^{\prime} / A}^{m}\right)\right) \otimes\left(\mathscr{H}_{k}^{3 r(g-1)}\right)^{-m a}
$$

is big (cf. [25, (5.1.1) (d)]) and hence $\alpha^{\prime}{ }_{*}\left(\omega_{X^{\prime} / A}^{m}\right) \otimes\left(\mathscr{H}_{k}^{3 r(g-1)}\right)^{-m}$ is also big. Since $H_{k}$ is ample, $3 H_{k}$ is very ample. Let $C$ be a curve obtained by intersecting $g-1$ general elements in $\left|3 H_{k}\right|$ and $\mathscr{A}:=\left.\mathscr{H}_{k}^{3 r(g-1)}\right|_{C}$. Then

$$
\operatorname{deg} \omega_{C}=(g-1)\left(3 H_{k}\right)^{g} \quad \text { and } \quad \operatorname{deg} \mathscr{A}=3 r(g-1) H_{k} \cdot\left(3 H_{k}\right)^{(g-1)}=r \operatorname{deg} \omega_{C} .
$$

Let $Y=\left(\alpha^{\prime}\right)^{-1}(C)$ and $h=\left.\alpha^{\prime}\right|_{Y}: Y \rightarrow C$. If $\alpha$ is smooth in codimension one, then $\alpha^{\prime}$ is smooth in codimension one and so $h$ is smooth. Since

$$
\left(\alpha^{\prime}{ }_{*}\left(\omega_{X^{\prime} / A}^{m}\right) \otimes\left(\mathscr{H}_{k}^{3 r(g-1)}\right)^{-m}\right) \mid C=h_{*}\left(\omega_{Y / C}^{m}\right) \otimes \mathscr{A}^{-m},
$$

it follows that $h_{*}\left(\omega_{Y / C}^{m}\right) \otimes \mathscr{A}^{-m}$ is also big and hence ample. By [28, Proposition 4.1] (with $\delta=0, s=0)$, one has

$$
\operatorname{deg} \mathscr{A}<\operatorname{dim}(Y / C) \operatorname{deg} \omega_{C}=r \operatorname{deg} \omega_{C} .
$$

This is impossible and hence $\alpha$ is not smooth in codimension one.

Proof of Theorem 1.4. - Since $A$ is simple, by [27, 10.9] (cf. [25, Theorem 3.7]) any proper subvariety of $A$ is of general type. By (3.2) we may assume that $\operatorname{alb}_{X}: X \rightarrow A$ is surjective. Then by (3.4), we may assume that $\operatorname{alb}_{X}$ is smooth in codimension one (again using [27, 10.9] to see that every divisor is of general type).

Now let $X \rightarrow Z \rightarrow A$ be the Stein factorization of $\operatorname{alb}_{X}$. Then $Z \rightarrow A$ is smooth and hence étale in codimension one, so $Z$ is birational to an abelian variety. It follows that $Z$ is birational to $A$ and $\operatorname{alb}_{X}: X \rightarrow A$ is an algebraic fiber space. Since $X$ is of general type, $\left(\operatorname{alb}_{X}\right)_{*}\left(\omega_{X / A}^{m}\right)=\left(\operatorname{alb}_{X}\right)_{*}\left(\omega_{X}^{m}\right)$ is big for some $m>0$, but by (3.5) this is impossible.

\section{REFERENCES}

[1] Akyildiz E., CARrell J.B., Zeros of holomorphic vector fields and the Gysin homomorphism, in: Singularities, Part 1, Arcata, CA, 1981, in: Proc. Sympos. Pure Math., vol. 40, American Mathematical Society, Providence RI, 1983, pp. 47-54, MR713044 (85c:32020).

[2] Akyildiz E., CARrell J.B., Lieberman D.I., Zeros of holomorphic vector fields on singular spaces and intersection rings of Schubert varieties, Compositio Math. 57 (2) (1986) 237-248, MR827353 (87j:32086).

[3] Akyildiz E., Carrell J.B., Lieberman D.I., Sommese A.J., On the graded rings associated to holomorphic vector fields with exactly one zero, in: Singularities, Part 1, Arcata, CA, 1981, in: Proc. Sympos. Pure Math., vol. 40, American Mathematical Society, Providence, RI, 1983, pp. 5556, MR713045 (84i:14029).

[4] BAUM P., BOTT R., On the zeros of meromorphic vector-fields, in: Essays on Topology and Related Topics, Mémoires dédiés à Georges de Rham, Springer, New York, 1970, pp. 29-47, MR0261635 (41 \#6248). 
[5] BAum P., Botт R., Singularities of holomorphic foliations, J. Differential Geom. 7 (1972) 279-342, MR0377923 (51 \#14092).

[6] Bотт R., Vector fields and characteristic numbers, Michigan Math. J. 14 (1967) 231-244, MR0211416 (35 \#2297).

[7] CARrell J.B., Holomorphic one forms and characteristic numbers, Topology 13 (1974) 225-228, MR0348147 (50 \#645).

[8] Carrell J., Howard A., Kosniowski C., Holomorphic vector fields on complex surfaces, Math. Ann. 204 (1973) 73-81, MR0372262 (51 \#8478).

[9] CARrell J.B., Lieberman D.I., Holomorphic vector fields and Kaehler manifolds, Invent. Math. 21 (1973) 303-309, MR0326010 (48 \#4356).

[10] Carrell J.B., Lieberman D.I., Vector fields and Chern numbers, Math. Ann. 225 (3) (1977) 263273, MR0435456 (55 \#8416).

[11] Du Bois P., Complexe de de Rham filtré d'une variété singulière, Bull. Soc. Math. France 109 (1) (1981) 41-81, MR613848 (82j:14006).

[12] ELKIK R., Singularités rationnelles et déformations, Invent. Math. 47 (2) (1978) 139-147, MR501926 (80c:14004).

[13] ElKIK R., Rationalité des singularités canoniques, Invent. Math. 64 (1) (1981) 1-6, MR621766 (83a:14003).

[14] Fulton W., Intersection Theory, Ergebnisse der Mathematik und ihrer Grenzgebiete (3) (Results in Mathematics and Related Areas (3)), vol. 2, Springer-Verlag, Berlin, 1984, MR732620 (85k:14004).

[15] Griffiths P., HARRIS J., Algebraic geometry and local differential geometry, Ann. Sci. École Norm. Sup. (4) 12 (3) (1979) 355-452, MR559347 (81k:53004).

[16] GREEN M., LAZARSFELD R., Deformation theory, generic vanishing theorems, and some conjectures of Enriques, Catanese and Beauville, Invent. Math. 90 (2) (1987) 389-407, MR910207 (89b:32025).

[17] Guillén F., Navarro Aznar V., Pascual Gainza P., Puerta F., Hyperrésolutions cubiques et descente cohomologique, Lecture Notes in Mathematics, vol. 1335, Springer, Berlin, 1988, Papers from the Seminar on Hodge-Deligne Theory held in Barcelona, 1982, MR972983 (90a:14024).

[18] Hartshorne R., Algebraic Geometry, Graduate Texts in Mathematics, vol. 52, Springer, New York, 1977, MR0463157 (57 \#3116).

[19] Kawamata Y., Minimal models and the Kodaira dimension of algebraic fiber spaces, J. reine angew. Math. 363 (1985) 1-46, MR814013 (87a:14013).

[20] Kollár J., Mori S., Birational Geometry of Algebraic Varieties, Cambridge Tracts in Mathematics, vol. 134, Cambridge University Press, Cambridge, 1998, With the collaboration of C.H. Clemens and A. Corti, Translated from the 1998 Japanese original, MR1658959 (2000b:14018).

[21] KovÁCS S.J., Families over a base with a birationally nef tangent bundle, Math. Ann. 308 (2) (1997) 347-359, MR1464907 (98h:14039).

[22] KovÁcs S.J., A characterization of rational singularities, Duke Math. J. 102 (2) (2000) 187-191, MR1749436 (2002b:14005).

[23] KovÁcs, S.J., Spectral sequences associated to morphisms of locally free sheaves, in preparation.

[24] LUO T., ZHANG Q., Holomorphic forms on threefolds, math.AG/0304022, 2003.

[25] Mori S., Classification of higher-dimensional varieties, in: Algebraic Geometry, Bowdoin, 1985, Brunswick, Maine, 1985, in: Proc. Sympos. Pure Math., vol. 46, American Mathematical Society, Providence, RI, 1987, pp. 269-331, MR927961 (89a:14040).

[26] ReID M., Minimal models of canonical 3-folds, in: Algebraic Varieties and Analytic Varieties, Tokyo, 1981, in: Adv. Stud. Pure Math., vol. 1, North-Holland, Amsterdam, 1983, pp. 131-180, MR715649 (86a:14010).

[27] Ueno K., Classification Theory of Algebraic Varieties and Compact Complex Spaces, Lecture Notes in Mathematics, vol. 439, Springer, Berlin, 1975, Notes written in collaboration with P. Cherenack, MR0506253 (58 \#22062).

[28] VIEHWEG E., ZUO K., On the isotriviality of families of projective manifolds over curves, J. Algebraic Geom. 10 (4) (2001) 781-799, MR1838979 (2002g:14012).

$4^{e}$ SÉRIE - TOME $38-2005-\mathrm{N}^{\circ} 4$ 
[29] ZHANG Q., Global holomorphic one-forms on projective manifolds with ample canonical bundles, J. Algebraic Geom. 6 (4) (1997) 777-787, MR1487236 (99k:14071).

(Manuscrit reçu le 4 novembre 2004; accepté le 16 décembre 2004.)

\author{
Christopher D. HACON \\ University of Utah, \\ Department of Mathematics, \\ 155 South 1400 East, Room 233, \\ Salt Lake City, UT 84112, USA \\ E-mail: hacon@math.utah.edu \\ http://www.math.utah.edu/ hacon
}

Sándor J. Kovács

University of Washington,

Department of Mathematics,

354350, Seattle, WA 98195, USA

E-mail: kovacs@math.washington.edu

http://www.math.washington.edu/ kovacs 